\title{
Heating cancer stem cells to reduce tumor relapse
}

\author{
Pier Giuseppe Pelicci*, Paola Dalton and Roberto Orecchia
}

\begin{abstract}
Tumour relapse is believed to be caused by rare cancer-cells with stem-cell properties (cancer stem cells) that are intrinsically resistant to available treatments. The identification of novel strategies to increase their sensitivity has major clinical implications. Latest clinical trials have shown a positive antitumoral effect of hyperthermia in combination with chemotherapy or radiotherapy. In a recent paper, the combination of increased temperature at the tumour site, generated by laser treatment of intravenouslyinjected gold nanoshells, and ionizing radiations enhances radiosensitivity of cancer stem cells and tumor response. At the root of the success of hyperthermia in enhancing radio-sensitization of cancer stem cells is the inhibition of their capacity to repair DNA damage, affecting the survival rate of these cells.
\end{abstract}

\section{Background}

According to the cancer stem cell (CSC) hypothesis [1,2], tumorigenicity, the capacity to propagate a tumor, is not an intrinsic property of any tumor cell, but the defining function of a specialized cell-type, the CSC. Within individual tumors, only CSCs are able to reform a tumor after transplantion in mouse recipients. Notably, these tumors have the same cellular heterogeneity as the initial one, including both tumorigenic (CSCs) and nontumorigenic cells (the remaining cells). CSCs express some surface markers of the corresponding normal tissue stem cells, while the non-tumorigenic remaining cells express markers of progenitors or more differentiated cells.

The existence of a cell hierarchy within tumors is shown by CSCs' ability to reform biologically heterogeneous tumors also from single-cell transplantation, whereby a CSC generates, through a single asymmetric mitotic division, phenocopies of itself (self-renewal) and differentiated/non-tumorigenic tumor cells [3].

*Correspondence: piergiuseppe.pelicci@ifom-ieo-campus.it European Institute of Oncology, Department of Experimental Oncology, Via Adamello 16, 20139 Milan, Italy
While the CSC theory lacks definitive proof, it bears important implications for cancer treatment. Anti-cancer drugs have been selected in early clinical trials for their property to reduce tumor size, an effect that reflects their ability to kill the bulk of tumor cells, but not necessarily the rare CSCs. These might then become the cellular base of clinical relapse.

Drugs capable of reducing tumor size and producing clinical remission are available for many tumor types. Nonetheless, tumors eventually relapse in a large proportion of cases, even after long intervals. For instance, in mammary tumors relapse rate after chemotherapy and/ or radiotherapy remains very high (up to approximately $50 \%$ ) and may occur as late as 30 years after manifestation of disease. Thus, the initial treatment might have spared a small population of cells with the ability to survive for long periods of time and to re-initiate tumor growth; cells that, according to the CSC theory, would be, by definition, CSCs. Stem cells are generally resistant to cytotoxic agents, probably because they are usually non-proliferating cells. Are CSCs also chemo- and radio-resistant, thus surviving in vivo treatments and initiating tumor regrowth? If so, increasing CSC sensitivity to the available chemo/radiotherapy treatments becomes a priority.

\section{Article}

The work published by Atkinson and colleagues [4] demonstrates, in preclinical models of mammary tumors, that CSCs are, in fact, resistant to radiotherapy, and that their sensitivity dramatically increases with concomitant local hyperthermia.

Therapeutic hyperthermia involves heating tissues to temperatures around 41 to $45^{\circ} \mathrm{C}$. While its potential benefits in cancer treatment were already acknowledged 5,000 years ago [5], results from recent clinical trials are often controversial, perhaps because of inadequate delivery systems, lack of appropriate monitoring of the temperature reached by the tumor, or intrinsic limitations of conventional heating sources. Notably, a meta-analysis of more recent data from randomized clinical trials of breast cancer patients, supported by thorough thermometry documentation, demonstrated that hyperthermia improves significantly both local tumor control and survival after radiation therapy [6], suggesting that, if properly delivered, it is a potent radiation sensitizer. 
Atkinson and colleagues used an innovative protocol of therapeutic hyperthermia based on the use of silica core nanoshells associated with thermocouple measurements. Nanoparticles are known to passively concentrate in tumor tissues after intravenous injection, thus allowing enhanced delivery of anticancer agents to malignant cells. Those employed in this study were designed to absorb light in the near-infrared spectrum and then release energy in the form of heat, significantly increasing the intra-tumoral temperature.

The authors used two preclinical model systems of breast cancer: the mouse p53-null mammary tumor, obtained after transplantation of p53-null mammary cells, and xenotransplants of 'triple-negative' human mammary tumors. Tumor-bearing mice were treated with radiotherapy alone (single dose) or radiotherapy plus hyperthermia (concurrently). Local hyperthermia was induced by near-infrared illumination of the tumor 24 hours after intravenous injection of the nanoparticles, leading to an intra-tumoral temperature of $42^{\circ} \mathrm{C}$ within 5 minutes, which persisted for 20 minutes. Mice were sacrificed 2 to 3 days after treatment and tumors analyzed for their CSC content, both directly within the tumor (using markers to identify CSC-enriched populations) and indirectly by their ability to form spheroids in culture or tumors in recipient mice upon limiting-dilution transplantation.

\section{Viewpoint}

The different treatment modalities gave comparable results in both model systems. Radiotherapy alone induced an overall decrease in tumor size, although to varying degrees. However, regardless of the degree of volume reduction, radiotherapy alone increased the relative numbers of CSCs (from 30 to 150\%). Notably, tumors that re-formed after X-ray treatment (upon transplantation) were more undifferentiated tumors. Hyperthermia alone did not show significant effects on tumor size or frequency of CSCs. The combination of X-rays plus hyperthermia, however, caused a greater regression of tumor size than the ionizing-radiation treatment alone, and, remarkably, a significantly lower percentage of CSCs. Accordingly, after the combination treatment, re-growing tumors appeared more differentiated.

Thus, CSCs are resistant to X-rays and are selected during treatment in vivo. These results indirectly suggest that resistant CSCs are responsible for tumor re-growth after-treatment, or, at least, that the re-formed tumors tend to lose their most differentiated component. They also show that hyperthermia, although ineffective by itself, increases the effect of X-rays on tumor growth by increasing CSC sensitivity to them.
This study also provides insights into the molecular mechanisms underlying the sensitizing effect of hyperthermia. The cytotoxic effect of X-rays is mainly due to induction of DNA double-strand breaks. While it was already known that CSCs are resistant to X-rays due to their unique ability to repair DNA damage, the authors show that hyperthermia diminishes this ability. It is likely, therefore, that, in the presence of hyperthermia, X-rays induce excess DNA damage and CSC death. It was recently shown that inactivation of DNA repair pathways causes DNA-damage 'overloading' in leukemia stem cells [7], leading to their functional exhaustion and preventing the development of the tumor clone. Thus, a growing body of evidence links CSC inherent resistance to DNA damage to tumorigenicity and to their resistance to currently available cytotoxic drugs. The identification of treatment modalities, including hyperthermia, that counteract the intrinsic capacity of CSCs to survive DNA damage emerges as a new promising strategy to target tumor growth and prevent tumor relapse.

Abbreviations

CSC, cancer stem cell.

Competing interests

The authors declare that they have no competing interests.

Published: 6 May 2011

References

1. Reya T, Morrison SJ, Clarke MF, Weissman IL: Stem cells, cancer, and cancer stem cells. Nature 2001, 414:105-111.

2. Lapidot T, Sirard C, Vormoor J, Murdoch B, Hoang T, Caceres-Cortes J, Minden M, Paterson B, Caligiuri MA, Dick JE: A cell initiating human acute myeloid leukaemia after transplantation into SCID mice. Nature 1994, 367:645-648.

3. Cicalese A, Bonizzi G, Pasi CE, Faretta M, Ronzoni S, Giulini B, Brisken C, Minucci S, Di Fiore PP, Pelicci PG: The tumor suppressor p53 regulates polarity of self-renewing divisions in mammary stem cells. Cell 2009, 138:1083-1095.

4. Atkinson RL, Zhang M, Diagaradjane P, Peddibhotla S, Contreras A, Hilsenbeck SG, Woodward WA, Krishnan S, Chang JC, Rosen JM: Thermal enhancement with optically activated gold nanoshells sensitizes breast cancer stem cells to radiation therapy. Sci Trans/ Med 2010, 2:55ra79.

5. Breasted JH: The Edwin Smith surgical papyrus. In Therapeutic Heat and Cold. 2nd edition. Edited by Licht S. Baltimore: Waverly Press; 1930:196.

6. Vernon CC, Hand JW, Field SB, Machin D, Whaley JB, van der Zee J, van Putten WL, van Rhoon GC, van Dijk JD, González González D, Liu FF, Goodman P, Sherar M: Radiotherapy with or without hyperthermia in the treatment of superficial localized breast cancer: Results from five randomized controlled trials. International Collaborative Hyperthermia Group. Int J Radiat Oncol Biol Phys 1996, 35:731-744.

7. Viale A, De Franco F, Orleth A, Cambiaghi V, Giuliani V, Bossi D, Ronchini C, Ronzoni S, Muradore I, Monestiroli S, Gobbi A, Alcalay M, Minucci S, Pelicci PG: Cell-cycle restriction limits DNA damage and maintains self-renewal of leukaemia stem cells. Nature 2009, 457:51-56.

doi:10.1186/bcr2847

Cite this article as: Pelicci PG, et al.: Heating cancer stem cells to reduce tumor relapse. Breast Cancer Research 2011, 13:305. 